\title{
Redes públicas, descentralizadas y comunitarias de salud en Bolivia
}

\author{
Fernando Lavadenz, ${ }^{1}$ Nicole Schwab ${ }^{2}$ y Hendrik Straatman ${ }^{3}$
}

RESUMEN La Reforma de la Salud en Bolivia está basada principalmente en la descentralización y la equidad para mejorar la calidad y ampliar la cobertura de servicios de salud a las áreas rurales y pobres del país. Como experiencia piloto de reforma, el Ministerio de Salud y Previsión Social, el Gobierno Departamental y el Municipio de El Alto, suscribieron un acuerdo con una organización no gubernamental (ONG), a la cual transfirieron la gestión integral de una de las redes de servicios de salud del municipio. La transferencia se basó en un contrato de gestión con indicadores de proceso y resultados. Después de un año de funcionamiento, los resultados preliminares indican que, a través del acuerdo, se ha mejorado la calidad de la administración $y$ de los servicios, aumentado la cobertura y fortalecido los servicios de primer nivel de la red. La ocupación de camas, generalmente muy baja en hospitales de segundo nivel ( $43 \%$ en el departamento de La Paz, 1999), alcanzó $84 \%$ en el hospital de la red en su segundo semestre de operación; las consultas externas en la red aumentaron en $55 \%$ (83\% en el hospital y $18 \%$ en los centros del primer nivel) entre el primer semestre de 1999 y el primer semestre del 2000; los partos institucionales aumentaron en $41 \%$ y el porcentaje de partos atendidos en los centros del primer nivel aumentó de 5 a 9\% en el mismo período. Una reciente encuesta de satisfacción de los usuarios muestra que $87 \%$ de las personas atendidas en la red se sintieron altamente satisfechas con la atención recibida y que $75 \%$ de la población consultada recomendaría el servicio a otras personas. Estos datos no son típicos de los servicios de salud del país. Esta experiencia piloto sugiere que un cambio en la organización y administración de una red de servicios de salud con separación de funciones entre comprador y proveedor, combinado con una gerencia basada en resultados y la participación de la comunidad en el proceso, puede mejorar la calidad y eficiencia de los servicios, estimular la demanda y aumentar la satisfacción de los usuarios.

Palabras clave Reforma del sector de la salud, Bolivia.

Bolivia es uno de los países de América con peores indicadores de mortali-

Dirección General de Servicios de Salud, Ministerio de Salud y Previsión Social, La Paz, Bolivia. La correspondencia debe enviarse a Fernando Lavadenz a la siguiente dirección: Dirección General de Servicios de Salud, Ministerio de Salud y Previsión Social, Capitán Ravelo 2199, Zona Central, La Paz, Bolivia. Dirección electrónica: lavadenz@ ceibo.entelnet.bo

2 Dirección General de Servicios de Salud, Ministerio de Salud y Previsión Social, La Paz, Bolivia.

3 Embajada del Reino de los Países Bajos en Bolivia, La Paz, Bolivia. dad materna e infantil: 370 por 100000 nacidos vivos (1) y 67 por 1000 nacidos vivos (2), respectivamente. Las principales causas de muerte infantil son las diarreas e infecciones respiratorias agudas, así como la atención perinatal inadecuada (3). La mortalidad materna se debe principalmente a hemorragias e infecciones vinculadas al parto y a complicaciones de abortos inseguros (4).

En este marco, la Reforma de Salud en Bolivia presta especial atención al desarrollo de políticas destinadas a reducir las principales causas de mortalidad materna e infantil, para lo cual se siguen dos estrategias principales.

- Desde la perspectiva de la demanda, se busca garantizar que todas las mujeres tengan acceso a atención institucional durante el embarazo y el parto. Para este efecto, el Ministerio de Salud creó el Seguro Básico de Salud (SBS), lan- 
zado en 1996 como Seguro Nacional de Maternidad y Niñez y ampliado en 1999 como SBS. El SBS elimina la barrera económica que impedía a las mujeres pobres y a sus hijos el acceso a servicios de salud como el parto institucional y la atención obstétrica específica. El SBS garantiza un paquete de 75 prestaciones a toda la población, centrado en las causas principales de muerte materna e infantil y en las enfermedades transmisibles. El SBS presta servicios que pueden reducir la mortalidad y morbilidad principalmente entre los pobres. Los municipios financian el SBS con recursos de la coparticipación tributaria que reciben del Tesoro General de la Nación, en una cuenta especial exclusivamente reservada para el SBS. Además, el SBS contempla la inclusión de múltiples proveedores (ONG, Iglesia, Seguridad Social y privados) en la red de servicios de salud.

- Desde la perspectiva de la oferta, los procesos de reforma se concentran en la reorganización de los proveedores de servicios para mejorar la calidad, eficiencia y administración de los servicios de salud. Con esta estrategia se pretende lograr que el sistema esté más orientado hacia los resultados y hacia las demandas de la población, con el fin de aumentar la cobertura de los servicios y la satisfacción de los usuarios. Con este fin, se inició la experiencia piloto de El Alto, que, sobre la base de un contrato de gestión, transfirió la gestión integral de una red de servicios de salud a una organización no gubernamental (ONG) sin fines de lucro.

\section{Mejorar la calidad y eficiencia de los servicios de salud}

La experiencia piloto tenía que tomar en cuenta varios factores que actualmente obstaculizan el buen funcionamiento del sistema público de salud del país. Primero, el sistema público boliviano, que es responsable del $85 \%$ de los servicios de salud en Bolivia, no está orientado ni a la eficiencia ni a las demandas de la población. Segundo, la política de descentralización del sector público de la salud en años anteriores fraccionó las responsabilidades entre los diferentes niveles de Gobierno. El proceso empezó en 1994 con las leyes de Participación Popular y de Descentralización Administrativa, para llegar hoy a la siguiente distribución de responsabilidades: la propiedad de los establecimientos de salud, el mantenimiento de la infraestructura e inversión y el financiamiento del SBS se descentralizaron al municipio; la administración de los recursos humanos del sistema público de salud pasó a los Servicios Departamentales de Salud (SEDES), y la operativización local de las políticas de salud nacionales quedó en manos de los recién creados Distritos de Salud, dependientes del Servicio Departamental de Salud. Sin embargo, debido a esta distribución de responsabilidades se presentaron las siguientes dificultades:

a) Ausencia de responsabilización. La distribución imprecisa de responsabilidades llevó a una duplicación de funciones.

b) Ausencia de autonomía de gestión e incentivos. El personal de salud del sistema público es nombrado por el nivel departamental y responde generalmente a criterios de selección políticos. No existen indicadores de necesidad para la distribución de los recursos humanos. Además, no hay diferencias de remuneración en los diferentes niveles de gestión y no existen incentivos para estimular la producción ni la demanda.

c) Ausencia de autonomía financiera. Antes de la emisión de un reciente Decreto Supremo que otorga mayor autonomía financiera a los servicios de salud, los recursos generados por el establecimiento eran generalmente depositados en la cuenta municipal y solo se reinvertían parcialmente en el establecimiento.

d) Prioridades ambiguas. Los municipios son responsables por la in- versión en infraestructura y equipamiento, pero muchos de ellos dan prioridad a otros sectores para la inversión de sus recursos y cuando invierten en salud no se coordinan con el nivel departamental para disponer de recursos humanos que operen en las infraestructuras creadas o equipadas.

e) Ausencia de integración de la red de servicios. Teóricamente, el Distrito de Salud tiene a su cargo toda la red de servicios públicos, pero en la mayoría de los casos es responsable solamente de los servicios del primer nivel y no tiene la autoridad, capacidad o reconocimiento suficientes para negociar con los hospitales de segundo y tercer nivel el desarrollo de redes de referencia y contra-referencia eficaces. Una de las consecuencias es que el hospital, en la mayoría de los casos, se encuentra prestando servicios de atención básica que deberían ser resueltos en los centros de primer nivel de la red.

Para resolver estos problemas, la Unidad de Reforma de Salud del Ministerio de Salud y Previsión Social (MSPS) lanzó la iniciativa "Redes descentralizadas, públicas y comunitarias", que se inspiró en experiencias de otros países, principalmente en algunos aspectos del Sistema Nacional de Salud del Reino Unido, e incorporó un fuerte enfoque obstétrico-neonatal, así como la participación de la comunidad en todos los aspectos de la red.

Al mismo tiempo que el MSPS estaba desarrollando esta iniciativa, un distrito de salud del municipio de El Alto estaba cambiando su modelo de organización, lo que lo convirtió en un candidato ideal para poner en marcha el nuevo modelo.

\section{Hacia las redes descentralizadas, públicas y comunitarias}

El Alto es la cuarta ciudad de Bolivia, con una población estimada de 568922 habitantes en el año 2000 (5) y 
una tasa de crecimiento anual de 9,4\% (6), debida principalmente a la migración hacia la ciudad desde el altiplano rural. La población de El Alto es joven, con un $60 \%$ entre 14 y 30 años, principalmente bilingüe, y la familia media tiene 4,4 miembros. La tasa de pobreza es muy alta: se estima que 70 a $73 \%$ de la población vive en condiciones de pobreza y un $78 \%$ no tiene acceso a uno o más servicios básicos.

A pesar de estos indicadores socioeconómicos preocupantes que indican que existen altos niveles de necesidad, El Alto tiene una muy baja capacidad de oferta de servicios de salud (cuadro 1) y la demanda de servicios de salud también es muy baja, con una tasa media de ocupación de camas de 37,3\% en El Alto, comparada con $43,0 \%$ en Bolivia (7).

En este marco, la red descentralizada, pública y comunitaria que se constituyó en El Alto y que es el objeto de este artículo atiende a un cuarto de la población del municipio e incluye un hospital de segundo nivel con las cuatro especialidades básicas (pediatría, ginecología, cirugía y medicina interna) y ocho centros de salud de primer nivel.

\section{Fase 1. La red dentro del sistema público (hasta julio de 1999)}

Inicialmente, el hospital y los ocho centros de salud de la red estaban bajo administración pública, enfrentando los problemas descritos. Es importante notar que este distrito se benefició de un apoyo internacional poco común en el resto del país. La cooperación holandesa invirtió alrededor de US\$ 7 millones en la organización de un sistema de atención primaria con ocho centros de salud. El antiguo hospital público tenía 33 camas y los centros de salud tenían en total 8 camas de maternidad. A pesar de la inversión holandesa y del Banco Mundial para equipamiento del hospital, la demanda de servicios seguía siendo muy baja: los centros atendían diariamente solo 6 horas y tenían una tasa de ocupación de camas muy baja.

\section{Fase 2. Transferencia de la gestión del hospital (agosto de 1999 a enero de 2000)}

Con el objetivo de mejorar los servicios, la gestión del hospital fue transferida a una ONG sin fines de lucro. La capacidad del hospital y su infraestructura fueron ampliados con un incremento inicial de 33 a 65 camas. La transferencia se basó en un contrato de gestión entre la ONG y el municipio. Sin embargo, este contrato no fijaba indicadores de proceso o resultado, ni metas, objetivos claros o incentivos para el cumplimiento de políticas nacionales como el SBS. Además, la responsabilidad de la gestión de los recursos humanos se quedó en manos del SEDES. Así que era difícil para la ONG ser responsable de la producción de servicios.
Por otra parte, el hospital transferido seguía una política de auto-sostenibilidad financiera, objetivo que chocaba contra el SBS, dado que, con el Seguro, el hospital recuperaba solamente el costo marginal de las prestaciones. Sin el SBS, el hospital podía cobrar precios por encima de las tarifas del Seguro, permitiéndole mayor recuperación de costos. El SBS se basa no solamente en costos marginales, sino en costos marginales medios, dado que no diferencia tarifas entre proveedores de diferentes niveles de complejidad, poniendo a los hospitales de mayor complejidad en una posición de desventaja frente a los centros de salud. La implementación del SBS en el hospital llevó a un aumento drástico de la demanda de las prestaciones incluidas en el SBS. Para responder a esta demanda, el hospital aumentó el número de camas disponibles una segunda vez, de 65 a 110. Sin embargo, estas medidas no compensaron el aumento de demanda y, preocupado por la baja recuperación de costos, el hospital limitó las atenciones del SBS a un máximo de 30 consultas externas por día y 35 camas disponibles.

Un problema adicional generado por la transferencia del hospital a la ONG fue la disminución de la demanda de consultas externas en los centros de primer nivel. Debido a la ausencia de un buen sistema de referencia, los pacientes llegaban directamente al hospital, generando una sobrecarga de consultas que hubieran podido ser resueltas en los centros

CUADRO 1. Infraestructura e indicadores de recursos humanos. Bolivia, 1998 y 2000

\begin{tabular}{|c|c|c|c|c|c|}
\hline & $\begin{array}{c}\text { Médicos } \\
\text { No. (por } 10 \text { 000) }\end{array}$ & $\begin{array}{c}\text { Enfermeras } \\
\text { No. (por } 10 \text { 000) }\end{array}$ & $\begin{array}{c}\text { Enfermeras } \\
\text { auxiliares } \\
\text { No. (por } 10 \text { 000) }\end{array}$ & $\begin{array}{c}\text { Camas de } \\
\text { hospital }^{\mathrm{a}} \\
\text { No. }(\text { por } 10000)\end{array}$ & $\begin{array}{l}\text { Población } \\
\text { (año 2000) }\end{array}$ \\
\hline Bolivia & $2398(2,9)$ & $1136(1,4)$ & $3905(4,7)$ & $11583(13,9)$ & 8328700 \\
\hline La Paz (departamento) & $730(3,0)$ & $283(1,2)$ & $965(4,0)$ & $3395(14,1)$ & 2406377 \\
\hline La Paz (municipio) & $503(5,0)$ & $205(2,0)$ & $488(4,9)$ & $2340(23,3)$ & 1004437 \\
\hline El Alto & $116(2,0)$ & $32(0,6)$ & $125(2,2)$ & $423(7,4)$ & $568922^{b}$ \\
\hline
\end{tabular}

Fuente: Servicio Nacional de Información en Salud, 1998, e Instituto Nacional de Estadísticas, 2000.

a Estas cifras incluyen a las camas de todos los sectores (público, privado, seguridad social, ONG e lglesia).

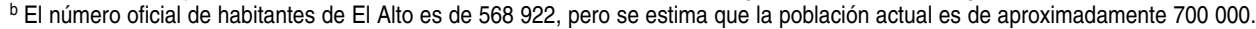


de primer nivel y dejaban a estos infrautilizados.

\section{Fase 3. La red descentralizada, pública y comunitaria (desde febrero de $\mathbf{2 0 0 0}$ hasta el presente)}

Un análisis del primer semestre de operaciones del hospital demostró que el contrato de gestión inicial no respondía a los problemas identificados en el análisis de la situación del sistema público de salud del país. La integración entre niveles de atención era insuficiente y era necesario desarrollar un sistema eficaz de referencia y contra-referencia en la red, así como introducir metas de gestión y un foro para la definición de políticas locales de salud. La autonomía financiera era parcial y la ausencia de responsabilidad llevó a un sistema de salud que no podía garantizar la puesta en práctica de las políticas nacionales.

Entonces, en el Distrito I de Salud de El Alto se desarrolló e implementó la iniciativa "Red descentralizada, pública y comunitaria", basada en los conceptos siguientes:

1. El comprador. Considerando el sistema de descentralización fraccionada establecido en el país, el comprador tenía que ser un "comité de compra" que pudiera reunificar a los diferentes actores dueños de los recursos, (municipio y SEDES). Se decidió que también se integraran en el comité de compra (llamado "Comité de Contrato de Gestión") dos representantes de la comunidad para incorporar un enfoque hacia la demanda y garantizar que la planificación respondería a las necesidades de la población.

2. El proveedor. Para integrar los servicios de salud, el proveedor no podía ser un hospital sin conexión con el primer nivel y se decidió que fuese la red de servicios de primer y segundo nivel del Distrito. Esta organización permite aumentar la relación costo-efectividad a través del fortalecimiento del primer nivel de atención y del desarrollo de un sistema de referencias y contrareferencias eficaces y eficientes entre el primer nivel y el hospital, que descargue a este de las consultas de primer nivel.

3. El contrato de gestión. Por primera vez en Bolivia, un comprador (el Comité de Contrato de Gestión) firmaba con el proveedor de servicios de salud un contrato de gestión con metas de resultados y procesos. Además, a través del contrato de gestión, se transfirió al proveedor la gestión integral de todos los recursos (físicos, humanos y económicos) del conjunto de establecimientos de la red.

\section{Cambios en la gestión producidos por el nuevo sistema}

La responsabilidad del prestador por la gestión de la totalidad de los recursos, el enfoque de gerencia por resultados definido por el contrato de gestión y la participación de la comunidad en todas las instancias determinadas por el sistema facilitaron importantes cambios en la red. Los principales fueron los siguientes: 1) se hizo más eficiente la gestión de recursos humanos mediante la generación de incentivos y capacitación para un cambio de mentalidad; 2) se implementó una oficina de defensa del cliente; 3) se mejoró la admisión y el sistema de orientación a los usuarios con 8 horas de apertura diaria para consultas externas y la distribución de fichas para reducir los tiempos de espera; 4) se desarrollaron normas y protocolos de atención para todas las prestaciones ofertadas en la red; 5) se transfirió a terceros la gestión de lavandería, alimentación y seguridad, y 6) se centralizaron los servicios de laboratorio y adquisición y almacenamiento de medicamentos para toda la red.

Estos cambios no hubieran sido posibles en un entorno diferente del creado, que otorgó al Alcalde, al Concejo Municipal, a los gestores y a la comunidad poder para negociar con los profesionales y trabajadores de la salud, creando condiciones favorables para un mejor desempeño y mayor motivación laboral.

\section{Resultados preliminares}

Considerando el corto tiempo de funcionamiento de las fases 2 y 3 , no se pueden esperar cambios sustanciales, pero los resultados preliminares proporcionan una visión del potencial impacto de los cambios en la gestión de los servicios sobre la producción de la red.

Se seleccionaron tres indicadores para medir el impacto del modelo:

1. La tasa de ocupación de camas y la duración media de la estancia como indicadores de proceso, que nos dan conjuntamente una idea de la eficiencia hospitalaria en la oferta de servicios.

2. Las consultas externas como indicador de demanda, debido a la baja demanda existente previamente en El Alto.

3. Los partos institucionales como indicador de resultado relacionado directamente con la mortalidad materna (8), que puede medir indirectamente el impacto.

Los cuadros 2 a 4 presentan los indicadores seleccionados en los semestres de las tres fases: enero a junio de 1999; agosto de 1999 a enero de 2000 y febrero a julio de 2000. No se toman en cuenta los datos del mes de julio de 1999 porque fue un mes de transición en la implantación del modelo, durante el cual el hospital solo recibió emergencias.

La tasa de ocupación de camas y la estancia media en el hospital aumentaron de $74 \%$ y 2,39 días, respectivamente, en la fase 1, a $84 \%$ y 3,67 días en la fase 3. Este incremento de $14 \%$ en la ocupación de camas ocurrió a pesar 
CUADRO 2. Evolución de la tasa de ocupación de camas y de la duración media de la estancia. El Alto, Bolivia, 1999-2000

\begin{tabular}{|c|c|c|c|c|c|c|}
\hline & \multicolumn{2}{|c|}{ Hospital } & \multicolumn{2}{|c|}{ Centros } & \multirow{2}{*}{\multicolumn{2}{|c|}{$\begin{array}{l}\text { Tasa de ocupación } \\
\text { (cambio porcentual) }\end{array}$}} \\
\hline & \multirow{2}{*}{$\begin{array}{c}\text { Tasa de ocupación }{ }^{a} \\
(\%)\end{array}$} & \multirow{2}{*}{$\begin{array}{l}\text { Estancia media } \\
\text { (días) }\end{array}$} & \multirow{2}{*}{$\begin{array}{c}\text { Tasa de ocupación }{ }^{a} \\
(\%)\end{array}$} & \multirow{2}{*}{$\begin{array}{c}\text { Estancia media }{ }^{b} \\
\text { (días) }\end{array}$} & & \\
\hline & & & & & Hospital & Centros \\
\hline Fase 1 & 74 & 2,39 & 10,7 & 0,97 & $-1^{c}$ & $142^{c}$ \\
\hline Fase 2 & 73 & 2,49 & 25,9 & 1,71 & $13^{d}$ & $-58^{d}$ \\
\hline Fase 3 & 83 & 2,65 & 11,0 & 0,96 & $12^{e}$ & $3^{e}$ \\
\hline $\begin{array}{l}\text { Fuente: } \\
\text { a Tasa de } \\
\text { b Tiempo } \\
\text { y gineco } \\
\text { c Entre las } \\
\text { d Entre las } \\
\text { e Entre las }\end{array}$ & $\begin{array}{l}\text { nal de Información en Salu } \\
\text { camas calculada como d } \\
\text { edia calculado como días- } \\
\text { tricia, que contaban con un }\end{array}$ & $\begin{array}{l}\text { lospital Municipal Boliv } \\
\text { ama disponibles sobre } \\
\text { ocupados sobre el tot } \\
\text { de } 30 \text { camas en la fas }\end{array}$ & $\begin{array}{l}\text { cupados. } \\
\text { os datos del hospital toman } \\
\text { has en las fases } 2 \text { y } 3 \text {. }\end{array}$ & uenta solamente la inf & os servicios & dicina interna \\
\hline
\end{tabular}

del aumento del número de camas de 33 a 110. Los datos agregados indican que gran parte del aumento de la tasa de ocupación de camas podría explicarse por el aumento de la duración media de la estancia. Sin embargo, los indicadores desagregados por servicio muestran resultados diferentes (cuadro 2). De las 110 camas del nuevo hospital, 28 están en el servicio de pediatría y 33 en traumatología, dos servicios que no existían en el hospital en la fase 1. Por lo tanto, el análisis se limitó a las camas de los servicios de ginecología y obstetricia y medicina interna. Combinando los indicadores de estos dos servicios, la tasa de ocupación aumentó de $74 \%$ en la fase 1 a $83 \%$ en la fase 3 y la estancia media aumentó de 2,39 a 2,65 días. En los centros de primer nivel, el incremento de la tasa de ocupación de camas en la fase 2 se puede atribuir a la duplicación de la duración media de la estancia, lo cual indica que no hubo cambios significativos en el número de pacientes ingresados en los centros de primer nivel en los tres períodos.

Las consultas externas en la red aumentaron en $55 \%$ entre las fases 1 y 3 : $83 \%$ en el hospital y $18 \%$ en los centros de salud (cuadro 3). Es interesante observar que entre las fases 1 y 2 , las consultas externas disminuyeron en $13 \%$ en los centros de primer nivel, evidenciando un cambio desde los centros hacia el hospital que refleja la "competencia" que existía entre ambos.

CUADRO 3. Evolución del número de consultas externas. El Alto, Bolivia, 1999-2000

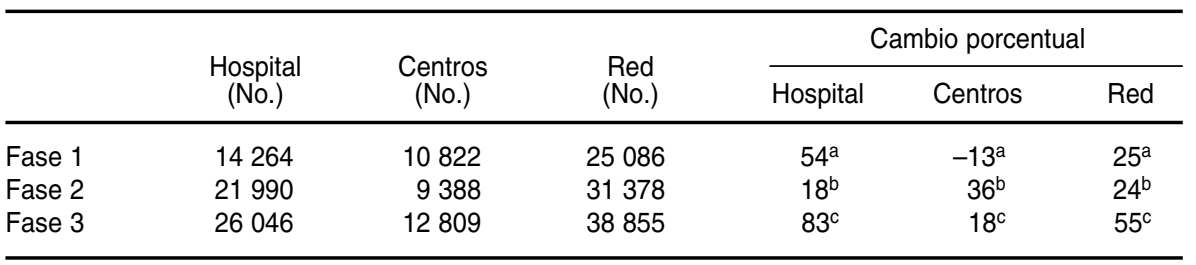

Fuente: Servicio Nacional de Información en Salud, 1999-2000.

a Entre las fases 1 y 2 .

${ }^{b}$ Entre las fases 2 y 3 .

${ }^{c}$ Entre las fases 1 y 3.

CUADRO 4. Evolución del número de partos según el lugar donde ocurrieron. El Alto, Bolivia, 1999-2000

\begin{tabular}{lcccccccc}
\hline & & & & & \multicolumn{4}{c}{ Cambio porcentual } \\
\cline { 6 - 9 } & Domicilio & Centros & Hospital & Red & Domicilio & Centros & Hospital & Red \\
\hline Fase 1 & 108 & 61 & 1083 & 1252 & $-11^{\mathrm{a}}$ & $33^{\mathrm{a}}$ & $27^{\mathrm{a}}$ & $24^{\mathrm{a}}$ \\
Fase 2 & 96 & 81 & 1375 & 1552 & $-27^{\mathrm{b}}$ & $86^{\mathrm{b}}$ & $12^{\mathrm{b}}$ & $14^{\mathrm{b}}$ \\
Fase 3 & 70 & 151 & 1545 & 1766 & $-35^{\mathrm{c}}$ & $148^{\mathrm{c}}$ & $43^{\mathrm{c}}$ & $41^{\mathrm{c}}$ \\
\hline
\end{tabular}

Fuente: Servicio Nacional de Información en Salud, 1999-2000.

${ }^{a}$ Entre las fases 1 y 2.

${ }^{\mathrm{b}}$ Entre las fases 2 y 3 .

${ }^{c}$ Entre las fases 1 y 3.

Considerando que el parto institucional es una de las políticas centrales del MSPS, el incremento de $41 \%$ en este indicador en un período tan corto es un dato alentador ${ }^{4}$. Los partos a domi-

\footnotetext{
4 Los datos se presentan en números absolutos y no en coberturas porque, a pesar de que el Distrito cubre una población estimada de 125185 (datos de diciembre de 1999), hay habitantes de otros distritos de El Alto y de áreas rurales vecinas que buscan atención en la red.
}

cilio disminuyeron entre las fases 1 y 3 , al mismo tiempo que aumentaron los partos en el hospital y en los centros de primer nivel, lo cual refleja un cambio acorde con la política nacional de partos institucionales y un fortalecimiento del primer nivel de atención dentro de la red de servicios (cuadro 4).

Para anular el incremento de la demanda atribuible al crecimiento de la población o a la implementación del 


\begin{tabular}{|c|c|c|c|c|c|c|c|c|c|c|c|c|}
\hline & & & & & & & & & \multicolumn{4}{|c|}{ Cambio porcentual } \\
\hline & \multicolumn{2}{|c|}{ Partos (No.) } & \multicolumn{2}{|c|}{$\begin{array}{l}\text { Ocupación de } \\
\text { camas (\%) }\end{array}$} & \multicolumn{2}{|c|}{$\begin{array}{l}\text { Estancia media } \\
\text { (días) }\end{array}$} & \multicolumn{2}{|c|}{$\begin{array}{c}\text { Camas de } \\
\text { hospital (No.) }\end{array}$} & \multicolumn{2}{|c|}{ Partos } & \multicolumn{2}{|c|}{$\begin{array}{l}\text { Ocupación de } \\
\text { camas }\end{array}$} \\
\hline & Red & $\begin{array}{l}\text { Distrito } \\
\text { V-VI }\end{array}$ & Red & $\begin{array}{l}\text { Distrito } \\
\text { V-VI }\end{array}$ & Red & $\begin{array}{l}\text { Distrito } \\
\mathrm{V}-\mathrm{VI}\end{array}$ & Red & $\begin{array}{l}\text { Distrito } \\
\text { V-VI }\end{array}$ & Red & $\begin{array}{l}\text { Distrito } \\
\text { V-VI }\end{array}$ & Red & $\begin{array}{c}\text { Distrito } \\
\text { V-VI }\end{array}$ \\
\hline Fase 1 & 1252 & 944 & 74 & 65 & 2,4 & 2,7 & 18 & 33 & $24^{b}$ & $9^{b}$ & $-1^{\mathrm{b}}$ & $18^{b}$ \\
\hline Fase 2 & 1552 & 1081 & 70 & 76 & 2,1 & 2,8 & 33 & 33 & $14^{\mathrm{C}}$ & $5^{c}$ & $13^{c}$ & $1^{c}$ \\
\hline Fase 3 & 1766 & 1135 & 87 & 77 & 2,2 & 2,7 & 33 & 33 & $41^{d}$ & $14^{d}$ & $12^{d}$ & $19^{d}$ \\
\hline
\end{tabular}

Fuente: Servicio Nacional de Información en Salud, 1999-2000.

a Para el hospital se toman en cuenta solamente los datos del servicio de ginecología y obstetricia, con un total de 18 camas en la fase 1 y 33 en las fases 2 y 3 en la red.

${ }^{\mathrm{b}}$ Entre las fases 1 y 2.

c Entre las fases 2 y 3.

${ }^{d}$ Entre las fases 1 y 3 .

SBS, es necesario comparar la situación de la red con otro distrito de El Alto (el Distrito V-VI), en el cual no se han realizado cambios de organización ni de gestión (cuadro 5). Dado que el hospital del Distrito V-VI es una maternidad, solo se consideran los siguientes indicadores: número de partos y tasa de ocupación y estancia media en el servicio de ginecología y obstetricia. Tanto la Maternidad del Distrito V-VI como el hospital de la red cuentan con 33 camas para ginecología y obstetricia. Los indicadores muestran un significativo aumento de los partos institucionales en la red descentralizada, pública y comunitaria del Distrito I (41\%), en comparación con el Distrito V-VI (14\%). Además, la tasa de ocupación de camas alcanzó $87 \%$, comparado con $76 \%$ en el Distrito V-VI. Estos resultados muestran que las mejoras en el funcionamiento de la red no se pueden atribuir únicamente a un aumento de la demanda generada por el SBS o por el crecimiento de la población de El Alto.

Con respecto a la satisfacción de los usuarios atendidos en la red, una encuesta realizada por una empresa independiente reveló que $87 \%$ de los pacientes que acudían al hospital estaban satisfechos con la atención recibida y que $75 \%$ de los usuarios consultados recomendarían el servicio a otras personas. No existen encuestas similares realizadas previamente en alguna de las fases de desarrollo del hospital ni de otros Distritos de El Alto. El que se haya realizado esta refleja una vez más la iniciativa del modelo de gestión existente y el cumplimiento del contrato de gestión que establecía la necesidad de realizarla.

\section{Conclusiones}

Analizando la evolución de los indicadores presentados y considerando las mejoras logradas en calidad, se puede llegar a las siguientes conclusiones preliminares:

1. El SBS fue un factor muy importante para el aumento de cobertura en El Alto y en el Distrito I, pero se combinó de forma directa con un nuevo modelo de gestión que incrementó aun más la demanda de servicios.

2. Los cambios en la gestión de la red, por medio de instrumentos como el contrato de gestión, han permitido introducir cambios e incentivos para mejorar la eficiencia y calidad de los servicios y conseguir un aumento real de la producción.

3. Esto llevó al aumento de las tasas de ocupación de camas, que indica que en el hospital se están resolviendo ciertas necesidades previamente insatisfechas, y al aumento del número de consultas externas y de partos institucionales en la red, que re- fleja un aumento de la demanda de servicios de salud en la red.

4. La actual organización de la red ha permitido disminuir la "competencia" entre el primer y el segundo nivel de atención y desarrollar un enfoque de "complementariedad" con niveles de integración crecientes.

Queda por comprobar si estos cuatro puntos se confirman en años venideros, pero mientras tanto se puede concluir que es posible introducir incentivos de producción, mejorar la calidad de los servicios ofertados y estimular la demanda de servicios de salud gracias a un cambio drástico en la gestión de una red integral de servicios de salud a través de la separación de funciones entre comprador y proveedor y la firma de un contrato de gestión con metas de resultados y procesos. Considerando la importancia del parto institucional para reducir la mortalidad materna e infantil en Bolivia, el aumento de la demanda de servicios puede aumentar la cobertura y, de esta manera, reducir el riesgo de parto domiciliario sin atención institucional.

\section{Implicaciones futuras}

El modelo piloto puesto en práctica en El Alto ya se está reproduciendo en otras partes del país, con las corres- 
pondientes adaptaciones al contexto local. Aunque la red de El Alto sirve a una población urbana concentrada, el modelo es válido en áreas rurales de población dispersa porque su enfoque no se basa en la competencia entre servicios, sino en la mejora de la gestión a través de la separación de funciones.

En zonas rurales con población dispersa donde la red de servicios cubra a más de un municipio se puede pensar en la creación de mancomunidades de municipios para lograr un objetivo específico común. A su vez, estas mancomunidades podrían asociarse con el SEDES correspondiente para formar el comité de compra que tiene la función de comprar servicios al proveedor local.

El modelo implementado en El Alto es un acuerdo entre una entidad pública y una entidad privada, pero nuevos modelos que se están implementando en el país contemplan acuerdos públicos-públicos. En el modelo público-público, el distrito, entidad pública, está asumiendo el papel de pro- veedor para organizar y gestionar la red. Este modelo es más factible en el país que el modelo público-privado, dado el escaso desarrollo del sector privado y la debilidad de las ONG. Además, este modelo se beneficia de una mayor aceptación política. La debilidad del modelo público-público reside en la baja capacidad de gestión de los servidores públicos y en el riesgo de que se vea sujeto a presiones políticas.

Otro factor que se debe tomar en consideración es la probable profundización de la descentralización hacia los municipios, principal conclusión del Diálogo Nacional 2000, que consolida las demandas de la sociedad civil, de los gobiernos municipales y de las autoridades departamentales y nacionales. En el Diálogo Nacional se planteó la transferencia de los recursos humanos del sector público de la salud a los gobiernos municipales. El compromiso del gobierno nacional de seguir estas demandas implica una profundización de la descentralización con cambios importantes en la organiza- ción del sistema boliviano de salud y de las entidades que asumirán en el futuro las funciones de comprador $y$ proveedor de servicios. Estos cambios brindan la oportunidad de reformar el sistema de salud en el futuro, con una separación de funciones y un enfoque hacia la demanda.

La experiencia piloto de El Alto se ha propuesto iniciar una cuarta fase, orientada al desarrollo tecnológico de la red (cirugía ambulatoria, educación médica y otros), a la calidad y adecuación cultural (aseguramiento y mejora continua y medicina basada en la evidencia), y a la integración con un hospital de tercer nivel de atención y con múltiples prestadores. Esta experiencia puede constituir un ejemplo de la introducción de una gestión basada en los resultados y en la separación de funciones con una delimitación más clara de las responsabilidades para mejorar la eficiencia y calidad de los servicios con el objetivo final concreto de disminuir la mortalidad materna e infantil en el país.

\section{REFERENCIAS}

1. Instituto Nacional de Estadística. Encuesta Nacional de Demografía y Salud, 1994. La Paz: INE; Octubre, 1994.

2. Instituto Nacional de Estadística. Encuesta Nacional de Demografía y Salud, 1998. La Paz: INE; Diciembre, 1998.

3. Ministerio de Salud y Previsión Social. Situación de salud de la niñez boliviana frente al nuevo milenio, Bolivia 2000. La Paz: MSPS; 2000.

4. World Health Organization. Making pregnancy safer: a health sector strategy for re- ducing maternal and perinatal morbidity and mortality. Geneva: WHO; 2000 (WHO/RHR/ 00.6.).

5. Instituto Nacional de Estadísticas. Proyecciones de Población. [Sitio en Internet]. Disponible en: http://www.ine.gov.bo. Acceso el 20 de marzo del 2001.

6. Ministerio de Salud y Previsión Social. Plan Estratégico de Salud: Ciudad de El Alto, 1999. La Paz: MSPS; 1999.

7. Servicio Nacional de Información en Salud. Datos de 1999. [Sitio en Internet]. Disponible en: http://www.sns.gov.bo. Acceso el 20 de marzo del 2001.

8. Banco Mundial. Proyecto de Reforma de Salud. Informe No. 18980-BO. La Paz: Banco Mundial. 31 de Marzo de 1999.

Artículo recibido el 30 de noviembre de 2000. Aceptado para publicación, tras revisión, el 26 de febrero de 2001. 
ABSTRACT Health sector reform in Bolivia is based primarily on the principles of decentralization and equity, and with the objectives of improving quality and of expanding health services coverage in rural and low-income areas of the country. As an experiment in re-

Public, decentralized, and community health networks in Bolivia form, the Bolivian Ministry of Health and Social Welfare, the department of La Paz, and the municipality of El Alto signed an agreement with a nongovernmental organization (NGO), transferring to the NGO the overall management of one of the health services networks in El Alto. The transfer was based on a management contract that had process and outcome indicators for the network. A year after implementation began, the preliminary results suggest that through the agreement the quality of management and services has improved, health services coverage has expanded, and the network's primary care services have been strengthened. Bed occupancy rates are generally very low in secondary hospitals in Bolivia, with that figure being just $43 \%$ in the department of La Paz in 1999. However, in the second 6 months of operation of the El Alto network the occupancy rate for the network's hospital reached $84 \%$. Between the first 6 months of 1999 and the same period in 2000, outpatient consultations increased by $55 \%$ in the network (83\% in the hospital and $18 \%$ in the network's primary care centers). Over that same period, institutional deliveries increased by $41 \%$ and the percentage of deliveries in the primary care centers grew from $5 \%$ of the total to $9 \%$. A recent user survey found that $87 \%$ of the people receiving care in the network felt highly satisfied with the service they had received, and $75 \%$ of the persons surveyed said they would recommend the service to others. These are not typical data for health services in Bolivia. This pilot effort suggests that a change in the organization and management of a health services network, with a separation of the roles of purchaser and provider, combined with management based on results and with community participation in the process, can improve the quality and efficiency of those health services, stimulate demand for them, and increase user satisfaction.

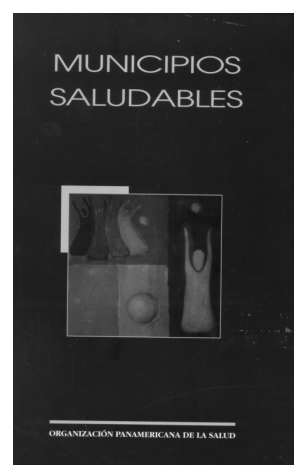

1997, 42 pp.

Código: CS 11

ISBN 927532060 8,

Precio: $\$ 10.00$

\section{PUBLICACIÓN DE LA OPS}

Municipios Saludables tiene el objetivo de atraer la creatividad y el compromiso individual y colectivo para crear espacios y estilos de vida saludables donde cada ciudadano tenga la posibilidad de gozar de todas las opciones vitales. El texto señala las amenazas para la salud y calidad de vida representadas por distintos factores que actúan sobre los entornos urbanos actuales; por ejemplo, el crecimiento caótico de las ciudades, el desarrollo industrial descontrolado, el número cada vez mayor de automóviles y la migración rural-urbana contribuyen a la formación de zonas marginales y a la proliferación de tugurios, a la contaminación ambiental, el hacinamiento y el aumento de los índices de enfermedades y violencia. El libro es de interés para todas las personas y sectores interesados en el desarrollo humano.

$$
\text { http://publications.paho.org • Fax: (301) 206-9789 • }
$$$$
\text { Correo electrónico:paho@pmds.com }
$$ 